\title{
Environment Topological Structure Recognition for Robot Navigation
}

\author{
Enver Sangineto ${ }^{1}$ and Marco R. Iarusso ${ }^{1,2}$ \\ 1 Centro di Ricerca in Matematica Pura ed Applicata (CRMPA), \\ 2 Dipartimento di Informatica e Automazione (DIA), \\ Universitá Roma 3, via della Vasca Navale 79, 00146, Rome, Italy
}

\begin{abstract}
Robot navigation using only abstract, topological information on the environment is strongly related to the possibility for a robot to unambiguously match information coming from its sensors with the basic elements of the environment. In this paper we present an approach to this challenging problem based on the direct recognition of the topological structure of the environment.
\end{abstract}

\section{Motivations and Goals}

In this paper we face the problem of robot navigation in an unknown indoor environment using information from an uncalibrated, single camera. In [10] robot navigation systems are categorized in systems based either on topological information (topological maps) or on metric information (metric maps). In the last two decades Computer Vision techniques have been frequently involved in the robot's self localization process, especially in conjunction with metric, a priori information. A typical vision-guided navigation system aims to recognize specific landmarks 2] whose exact position in the environment is known to the robot and allows it to self-localize. Some early systems use artificial landmarks, i.e., objects placed ad hoc in the environment for the recognition purpose, whose visual features (e.g., shape or color) facilitate their recognition. Examples of artificial landmarks are simple geometrical patterns [4] or bar codes 6].

On the other hand, natural landmarks can be any kind of objects belonging to the environment such as (in the indoor example): doors, windows, bulletin boards, etc.. Natural landmarks are less intrusive but usually more difficult to recognize. Dulimarta and Jain [5] use ceiling lights and door number plates as landmarks for self-localization. Kosaka and Kak [8] compare previously (handmade) 3D CAD models of the hallway environment with the input frames. Modeling with a Kalman filter the uncertainties in the position of the robot (influenced by the motion parameters) it provides a framework for the selection of the most likely positions of the landmarks in the image. A projective invariant technique is used by Wang and colleagues [9] for the robot self-localization task. Intersection points between straight vertical lines representing doors and the floor are used as features. A data base containing the projective invariants computed for the whole hallway is off-line built. On-line, the extraction of the intersection points 
from the camera frame is used to search for in the data base and to compute the robot position by means of an alignment method.

A common problem with all these approaches is the need of an exact a priori knowledge of the landmarks' positions with respect to a metric description of the robot's environment. An attempt to perform landmark-guided navigation in an unknown environment has been done by Trahanias et al. 11] who use landmarks for topological-based navigation. Nevertheless, the patterns of the landmarks they use (e.g., a box or a fire extinguisher fixed to the corridor's walls) need to be previously learnt by the system, and thus also in this case there is an (partial) a priori knowledge on the environment. Moreover, the approach is potentially unstable, because the objects selected as landmarks can change their positions.

In this paper we propose to directly recognize the different kinds of topological elements which characterize the robot's environment. Rather then trying to associate arbitrarily chosen objects or landmarks to specific environments, we propose to directly classify the sub-environments the robot meets during its navigation-discovery by the exploitation of their perspective appearance. We assume that the robot works in an hallway environment, hence the topological elements the system needs to distinguish are: straight corridor (the two corridor's walls in the proximity of the robot are not interrupted by corners or crossings: e.g., see Figure 1 (a)), left or right corner (the robot is next to a wall with the possibility to turn only on the left or only on the right, as in Figure 1 (c)), end of corridor (a wall is in front of the robot without corners), T-junction (a wall is in front of the robot with two corners) and crossing (as in Figure 1 (b)).

The recognition process is based in an incremental reconstruction of the main structural elements of the hallway, as they appear in a perspective view. First, the system performs a standard edge detection and polygonal approximation of the current camera frame. The segments extracted from the polygonal approximation are then used in order to find the z-axis vanishing point with a modified Hough Transform approach [1]. There is a large literature on vanishing point search techniques (see, for instance, [3]). Our aim here is not to propose a general-purpose vanishing point search method but to exploit the assumptions on the robot's environment (a corridor in which main information about the walls can be described using segments) to perform accurate and real-time computations. Once the vanishing point has been found in the image, we use a Hough-like approach to find the edges of the floor. Finally, we analyze the edges of the walls and of the floor in order to understand the topological configuration of the corridor. The computational complexity of each of the previous steps is at most squared with respect to the number of segments and the whole execution time takes only few second's fractions.

\section{Efficient Vanishing Point Search}

First of all we perform a standard edge detection and thinning process by using the Canny edge detector with Sobel $3 \times 3$ masks [7]. Then the edge points are grouped in lines (i.e. continuous sets of points in which each point is adja- 


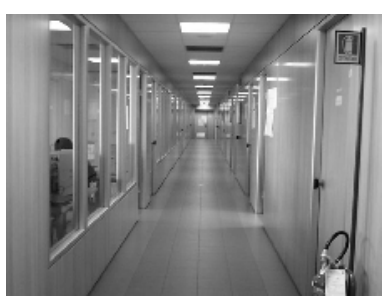

(a)

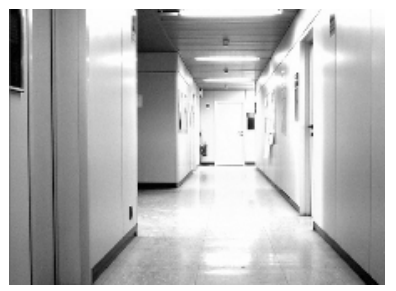

(b)

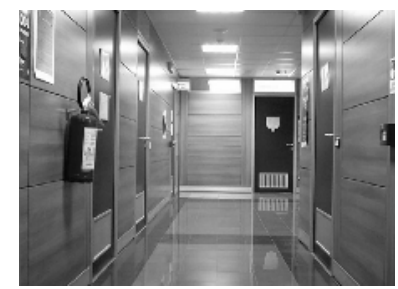

(c)

Fig. 1. Three different types of topological hallway environments: (a) a straight corridor, (b) a crossing and (c) a right corner.

cent to at most two other points) and the lines approximated using a standard split polygonal approximation method [7. The segments so obtained are then used as atomic elements of the subsequent elaborations. This leads to both save computational time (since the number of segments belongs to a lower order of magnitude with respect to the number of edge pixels) and reduce noise from data. In fact, since our scene understanding is based on the analysis of walls and walls' intersections, we do not deal with smoothed objects, thus ignoring human people and any other nonpolygonal object possibly present in the corridor.

The segments are represented by means of the tuple: $\left\langle\alpha, \rho, P_{1}, P_{2}\right\rangle$, where $\alpha$ and $\rho$ are, respectively, the angle of the normal and the distance of the straight line passing through the segments. The line is represented by the equation [7]: $\rho=x \cos \alpha+y \sin \alpha$, where the coordinate reference system is fixed in the bottom left corner of the image $I$ and $0 \leq \alpha<360,0 \leq \rho \leq R, R$ being the diagonal of $I$. Finally, $P_{1}=\left(x_{1}, y_{1}\right)$ and $P_{2}=\left(x_{2}, y_{2}\right)$ are the two endpoints of the segment.

Let $S$ be the set of all the segments. Using the values of the $\alpha$ parameter we can classify each segment as belonging to the set of vertical segments $V=$ $\left\{s \in S: s=\left\langle\alpha, \ldots>, \alpha \in\left[\right.\right.\right.$ th $\left.\left._{1}, t_{2}\right]\right\}$, horizontal segments $H=\{s: s \in$ $S: s=\left\langle\alpha, \ldots>, \alpha \in\left[t h_{3}, t h_{4}\right]\right\}$ and oblique segments $O=\{s: s \in S: s \notin$ $V \wedge s \notin H\}$, where $t h_{1}, t h_{2}, t h_{3}$ and $t h_{4}$ are prefixed thresholds (presently we set: $t h_{1}=-5, t h_{2}=5, t h_{3}=85, t h_{4}=95$ ). Figure 2 (a) shows the sets $V, H$ and $O$ extracted from the image of Figure 1 (b).

In the next step, we search for the z-axis vanishing point using the elements of $O$. For each couple of segments $s=<\alpha_{s}, \rho_{s}, \ldots>$ and $s^{\prime}=<\alpha_{s^{\prime}}, \rho_{s^{\prime}}, \ldots>$, $s, s^{\prime} \in O$, we compute the intersection point $P_{s s^{\prime}}$ between the two straight lines represented by $\alpha_{s}, \rho_{s}$ and $\alpha_{s^{\prime}}, \rho_{s^{\prime}}$ respectively. $P_{s s^{\prime}}$ is used to vote in a Hough accumulator $A$ of the same dimensions of $I$ which is initially set to zero $(A(x, y)=$ $0, \forall(x, y) \in I)$. Given the intersection point $P_{s s^{\prime}}$, the vote action is: $A\left(P_{s s^{\prime}}\right):=$ $A\left(P_{s s^{\prime}}\right)+|s|+\left|s^{\prime}\right|$, being $|x|$ the length of the segment $x$.

A common, well-known problem with Hough methods is the need to find a suitable grid size for the accumulator's representation [7. Too fine a grid can produce vote dispersion because different elements of the same object can vote in different accumulator's cells due to discretization and noise effects. On the other 
hand, too coarse a grid can produce merging of votes belonging to different objects in a unique accumulator's cell. We deal with this problem by using a fine representation (each cell of $A$ corresponds to a pixel in $I$ ) and then clustering the votes using a technique presented in [1]. The clustering is done as follows.

After that the voting operation has been executed for all pairs of oblique segments, $A$ represents the result of the voting process. Since lines which converge in a real image can spread their vote in different cells of the accumulator because discretization and noise effects, we want to find in $A$ the most "heavy" area, which represents the most likely position of the vanishing point. For this reason we scan $A$ using a squared mask $W_{2 l+1 \times 2 l+1}$. The size of $W$ should be chosen in order to let the window to include the expected area of vote dispersion (we set $l=3$ ). When $W$ is centered in $p$ we take into account only the votes in the neighborhood of $p$ contained in the mask $W$. Let us denote with $W(p)$ the set of all the nonzero cells of $A$ contained in the mask $W$ when it is centered on $p$. For a given set $W(p)$ we compute the "mass" $M(p)$ of $W(p)$, as the sum of the values of the elements of $W(p)$. The maximum found value $\bar{M}=\max _{p \in I} M(p)$ corresponds to the mass of the most likely area for the vanishing point and it can be computed scanning sequentially each row $y$ of $A$ and using a dynamic programming technique [1. Assuming (for simplifying reasons) to compute $M(p)$ for only those points far at least $l$ from the boarders of $I$, for a given point $p$ $(p=(x, y))$ the mass $M(p)$ is given by:

$$
M(x, y)=\left\{\begin{array}{lr}
\sum_{p_{1} \in W(x, y)} A\left(p_{1}\right), & x=l+1, \\
M(x-1, y)-\sum_{p_{1} \in W_{1}(x-1, y)} A\left(p_{1}\right)+\sum_{p_{1} \in W_{2 l+1}(x, y)} A\left(p_{1}\right), & x>l+1
\end{array}\right.
$$

where $W_{i}(p)$ represents the nonzero elements of the i-th column of the mask $W(p)$. In other words, for a given row $y$ of $A$, the mass can be computed using the base case of Equation (11) for the first cell and the inductive case for all the others (except the last $l$ cells on the right). If $\bar{p}=\arg \max _{p \in A} M(p)$, then the coordinates $(Z)$ of the z-axis vanishing point are given by the centroid of the elements of $W(\bar{p})$ :

$$
Z=\frac{\sum_{p_{1} \in W(\bar{p})} A\left(p_{1}\right) p_{1}}{\bar{M}}
$$

\section{$3 \quad$ Splitting Lines}

The next step in the perspective structure reconstruction is to find the 2 lines separating the floor from the lateral walls. Unfortunately we cannot simply clustering all the segments converging on the vanishing point $Z$ because these are spread all along the image. Nevertheless, we observe that the (ideal) splitting lines are close to the endpoints of a lot of vertical segments (e.g., the conjunctions of the doors with the floor) and horizontal segments (e.g., the points in which the tails are close to the walls). This information can be exploited in the searching process.

We use for this task a second Hough accumulator $A^{\prime}$ to represent the parameters $\alpha$ and $\rho$ of all the lines of the image $I . A^{\prime}$ is initially set to zero. 


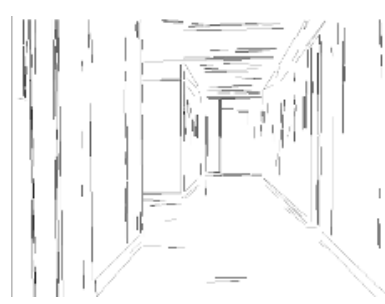

(a)

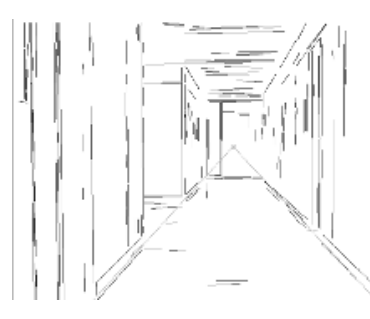

(b)

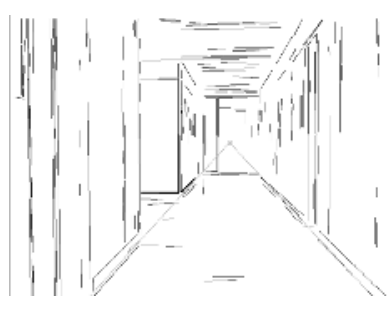

(c)

Fig. 2. The intermediate results of the recognition process. (a) The sets of segments $V$, $H$ and $O$ for the image of Figure 1 (b), each set represented with a different graylevel. (b) The same image after the splitting lines' extraction. (c) The $E_{1}$ type of topological edges' configuration recognized on the crossing scene.

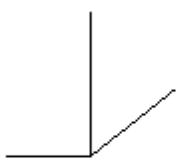

El

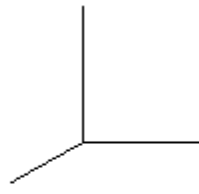

E2

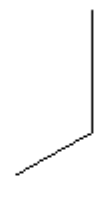

E3

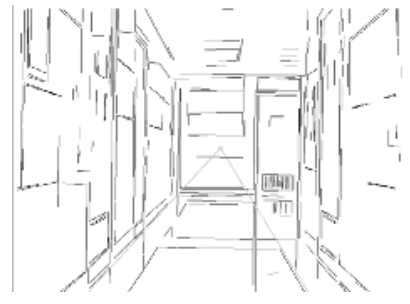

(b)

(a)

Fig. 3. (a) The three types of topological edges configurations (b) The example of Figure 1 (c) in which the right splitting line has been wrongly extracted (see the text).

For each $v \in V$ and each $o \in O\left(o=<\ldots, P_{1}^{o}, P_{2}^{o}>\right)$ we verify if $v$ and $o$ are close enough to be considered joined. More in details, for each endpoint $P^{v}=\left(x^{v}, y^{v}\right)$ of $v$ we first of all check if $x^{v} \in\left[x_{1}^{o}, x_{2}^{o}\right]$, being $P_{1}^{o}=\left(x_{1}^{o}, y_{1}^{o}\right)$ and $P_{2}^{o}=\left(x_{2}^{o}, y_{2}^{o}\right)$. If this is the case, we compute the distance $d\left(P^{v}, o\right)$ of the point $P^{v}$ from $o$. If $d\left(P^{v}, o\right)$ is smaller than a given threshold $t h_{5}$ (we set $t h_{5}=10$ ) then $v$ and $o$ are considered joined and the junction point $j_{o v}$ is computed: $j_{o v}=\left(x^{v}, y^{v}+\frac{d\left(P^{v}, o\right)}{2}\right)$ if $v$ is below $o$ and $j_{o v}=\left(x^{v}, y^{v}-\frac{d\left(P^{v}, o\right)}{2}\right)$ otherwise.

The point $j_{o v}$ is used together with the vanishing point $Z$ in order to compute the parameters $\left(\alpha\right.$ and $\rho$ ) of the line $L$ passing through $j_{o v}$ and $Z$. $L$ is a candidate splitting line and $\alpha$ and $\rho$ are used to increment the accumulator: $A^{\prime}(\alpha, \rho):=$ $A^{\prime}(\alpha, \rho)+1$. The same procedure is repeated also for horizontal segments.

The 2 maximum values in $A^{\prime}$ after the voting process are retrieved using the same technique described in the case of the vanishing point (Section 2). From now on we will indicate the bottom-left and bottom-right splitting lines respectively with $L_{1}$ and $L_{2}$. Figure 2 (b) shows an example. 


\section{Topological Edges Configuration}

The final step of the proposed incremental scene interpretation is the recognition of the type of topological environment (as they have been defined in Section 1) the robot is looking at by analyzing the information previously extracted. We observe that when a lateral wall is interrupted by another orthogonal corridor (because a crossing), the edges of the joining walls form the configuration $E_{1}$ shown in Figure 3 (a). Analogously, a corner is characterized by the edge configuration $E_{2}$ shown in Figure 3 (a). The end of corridor is characterized by two configurations of type $E_{2}$ (one on the left and the other on the right side of the hallway). A T-junction is characterized by two configurations of type $E_{3}$. Finally, in a straight corridor we cannot observe anyone of the abovementioned configurations (at least not in the proximity of the robot). Thus, the current topological environment can be distinguished by looking for the topological edges configuration in the segments' dispositions.

Before to analyze the segments' configurations we first arrange a more suitable representation of the sets of segments $S$. In the following we show the representation criteria and the edge configurations' search with respect to the splitting line $L_{1}$ omitting to show the analogous process for $L_{2}$. We use Boolean tables $T_{K}[x]\left(0 \leq x \leq x_{Z}\right.$, being $\left.Z=\left(x_{Z}, y_{Z}\right)\right)$ initially set to false. $T_{K}[x]=$ true means that there exists a segment $s$ of type $K$ sufficiently close to $L_{1}$ where $P=(x, y)$ is one of the endpoints of $s$.

For each element $v \in V\left(v=<\ldots, P_{1}, P_{2}>\right)$ let $P(P=(x, y))$ be the point with the lowest ordinate between $P_{1}$ and $P_{2}$. We check that $P$ is above $L_{1}$, otherwise we discard $v$ : it is noise on the floor. If $v$ is sufficiently large and $P$ is sufficiently close to $L_{1}$ we set $T_{V}[x]:=$ true. Moreover, for each segment $h \in H$ $\left(h=<\ldots, P_{1}, P_{2}>\right.$ ) let $P=(x, y)$ be the point between $P_{1}$ and $P_{2}$ which is closer to $L_{1}$. If $h$ is sufficiently large and $P$ is sufficiently close to $L_{1}$ then we set either $T_{H_{L}}[x]:=$ true or $T_{H_{R}}[x]:=$ true depending on whether $h$ is located on the left or on the right side of $L_{1}$. Finally, if $o \in O\left(o=<\alpha, \rho, P_{1}, P_{2}>\right)$ we check that the distance of $Z$ from the line represented by $(\alpha, \rho)$ is no greater than $d=\sqrt{2\left(\frac{2 l+1}{2}\right)^{2}}$, where $2 l+1$ is the side of the window $W$ used in Section 2 in order to find $Z$. We discard $o$ if it is not convergent on $Z$. Furthermore, we discard $o$ if it is not sufficiently large and close to $L_{1}$. Without loss of generality, let $P_{1}=\left(x_{1}, y_{1}\right), P_{2}=\left(x_{2}, y_{2}\right)$ and $x_{2}>x_{1}$. We represent $o$ twofold: both by setting $T_{O_{D}}\left[x_{1}\right]:=$ true and by setting $T_{O_{U}}\left[x_{2}\right]:=$ true.

Once built $T_{V}, T_{H_{L}}, T_{H_{R}}, T_{O_{D}}$ and $T_{O_{U}}$ we start searching for the three kinds of topological edge configurations $E_{1}, E_{2}$ and $E_{3}$. Concerning $E_{1}$ the system works as follows. For each $x$ such that $T_{O_{D}}[x]=$ true, we check in a neighborhood of $x$ for the existence of $x^{\prime}$ and $x^{\prime \prime}$ such that $T_{V}\left(x^{\prime}\right)=$ true and $T_{H_{L}}\left(x^{\prime \prime}\right)=$ true. The presence of 3 segments $v$ (represented by $\left.T_{V}\left(x^{\prime}\right)=\operatorname{true}\right), o\left(T_{O_{D}}[x]=\right.$ true) and $h\left(T_{H_{L}}\left(x^{\prime \prime}\right)=\right.$ true $)$, close to each other and displaced the first vertically, the second (approximately) aligned with $L_{1}$ and the third on the left side of $L_{1}$ means that we have found a configuration of type $E_{1}$. An example of such a triple of segments is shown in Figure 2 (c). 
Looking for the $E_{2}$ type of configurations follows an analogous process. Let $x$ be such that: $T_{O_{U}}[x]=$ true and $\nexists w: T_{O_{U}}[w]=$ true $\wedge w>x$, where the second condition is important because we want that $x$ is the closest possible to $Z$ (no other oblique segments can be aligned with $L_{1}$ and closer to $Z$ if $x$ represents the point in which the floor meets a frontal wall). Finally, $E_{2}$ is recognized if we can find, $x^{\prime}$ and $x^{\prime \prime}$ in a neighborhood of $x$ such that: $T_{V}\left(x^{\prime}\right)=$ true and $T_{H_{R}}\left(x^{\prime \prime}\right)=$ true. $E_{3}$ is searched similarly.

As shown in the first part of this section, the combination of the possibly recognized types $E_{1}, E_{2}$ and $E_{3}$ allows the system to recognize the topological environment. In the case in which both $E_{1}$ and $E_{2}$ (or $E_{3}$ ) are recognized, the answer of the system is "crossing" (because $E_{1}$ is necessarily closer to the robot). If no type at all is recognized, the system's output is "straight corridor".

\section{$5 \quad$ Efficiency and Experimental Results}

For lack of space we have to omit a detailed analysis of the complexity of the whole recognition procedure. However, it is clear that each phase above presented, except the maxima searching in the Hough accumulators, have a computational cost at most squared with respect to the number of segments involved. For example, in Section 3 we look for a candidate splitting line by computing the intersection among all the possible pairs of segments $(v, o)$ and $(h, o)$, for each $v \in V, h \in h$ and $o \in O$. If $N=|S|$, this step is $\mathrm{O}\left(N^{2}\right)$. The dynamic programming technique used to search for the maximum in the Hough accumulator $A$ (Section 2) allows us to perform the search in $\mathrm{O}(M w)$, being $M=|A|=|I|$ and $w=2 l+1$. A similar result is obtained for the Hough accumulator $A^{\prime}$ in Section 3. As we can see, all the involved operations have a very low computational cost and thus can satisfy real-time requisites of a typical robot navigation task.

We have implemented and tested our system with a non-optimized Java code on a Pentium $4,2.66 \mathrm{GHz} \mathrm{CPU}$, with an average execution time of about 0.54 seconds per image. We have used as test data base 87 images randomly taken from 5 different corridors (3 of which are shown in Figure 1). No assumption has been done on the lighting conditions. In the $58.6 \%$ of the images the system has correctly recognized all the topological environments without any false positive or missing element. In the $78.2 \%$ of the images the system has correctly recognized the environment closest to the camera point of view. Errors are mainly due to the noise produced by the presence of strong lights on the ceiling or to mirror effects of the walls' edges on the floor (as in Figures 1 (c) and 3 (b)).

\section{Conclusions}

We have presented an approach to topological navigation for unknown, indoor environments based on the direct recognition of the topological structure of the corridor. By means of an incremental approach we reconstruct the structure 
of the walls present in the observed scene. For this reason, the method does not depend on the presence of specific objects (landmarks) whose position in the environment needs to be a priori known. Experimental results have shown real-time performances and good recognition skills.

\section{Acknowledgements}

We want to thank the students Elisabetta Cascino, Sergio Garofoli and Valentina Madonna for their precious help in developing and testing the second version of the system's prototype.

\section{References}

1. Anelli, M., Micarelli, A., And Sangineto, E. A deformation tolerant version of the generalized Hough transform for image retrieval. In Fifteenth European Conference on Artificial Intelligence (ECAI 2002), Lyon, France (2002).

2. Borenstein, J., Everett, H. R., And Feng, L. Navigating mobile robot: sensors and techniques. A. K. Peters, Ltd., Wellesley, MA, 1996.

3. Cantoni, V., Lombardi, L., Porta, M., And Sicard, N. Vanishing point detection: representation analysis and new approaches. In 11th International Conference on Image Analysis and Processing (ICIAP01) (2001), pp. 90-94.

4. Courtney, J. W., Magee, M. J., And Aggarwal, J. K. Robot guidanceusing computer vision. Pattern Recognition 17. No. 6 (1984), 585-592.

5. Dulimarta, H. S., And Jain, A. K. Mobile robot localization in indoor environment. Pattern Recognition 30. No. 1 (1997), 99-111.

6. Fiala, M. Linear markers for robot navigation with panoramic vision. In 1st Canadian Conference on Computer and Robot Vision (CRV04) (2004).

7. Forsyth, D. A., And Ponce, J. Computer Vision: A Modern Approach. Prentice Hall, 14 August, 2003, ISBN: 0130851981, 2003.

8. Kosaka, A., And KaK, A. C. Fast vision-guided robot navigation using modelbased reasoning and prediction of uncertainties. CVGIP 56. No. 3 (1992), 271-329.

9. Lee, W. H., Roh, K. S., And Kweon, I. S. Self-localization of a mobile robot without camera calibration using projective invariants. Pattern Recognition Letters 21 (2000), 45-60.

10. Thrun, S. Learning metric-topological maps for inddor mobile robot navigation. Artificial Intelligence 99 (1998), 21-71.

11. Trahanias, P. E., Velissaris, S., and Orphanoudakis, S. C. Visual recognition of workspace landmarks for topological navigation. Autonomous Robots 7 (1999), 143-158. 